In der Rubrik „Literatur kompakt" werden die wichtigsten Originalarbeiten aus der internationalen Fachliteratur referiert.

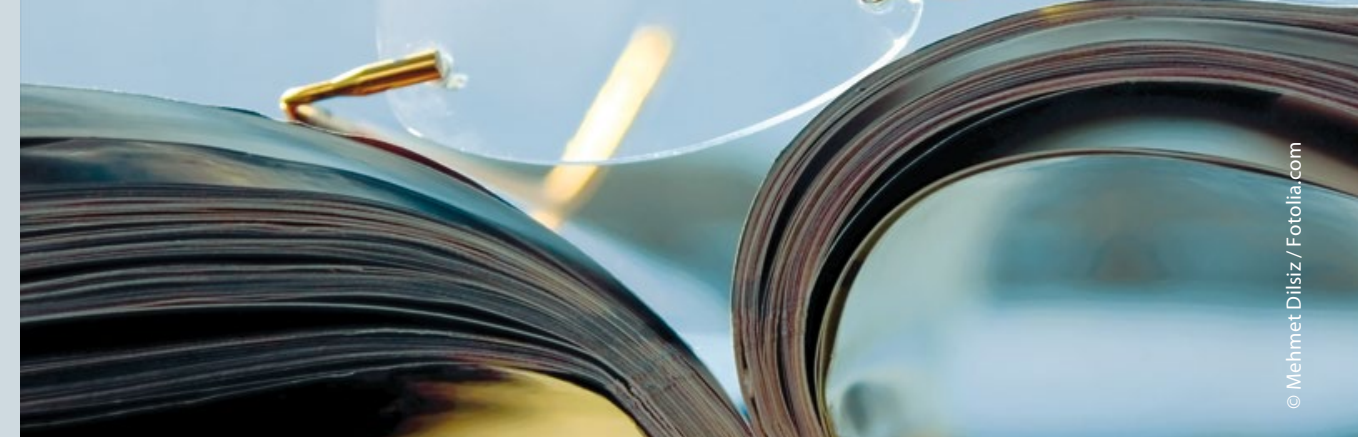

\section{Nerven orten: Wer schallt, der findet}

\section{Periphere Nervenblockaden gelingen häufiger, wenn die Suche nach den zu betäubenden Strukturen ultraschallgesteuert verläuft. Die elektrische Stimu- lation als Suchverfahren weist vergleichsweise geringere Erfolgsraten auf.}

m Zuge einer systematischen Übersichtsarbeit haben die britischen Anästhesisten Shilpa Munirama und Graeme McLeod 23 randomisierte und kontrollierte Studien metaanalysiert, an denen insgesamt 2.125 Patienten beteiligt gewesen waren. Bei den untersuchten Verfahren handelte es sich in 15 Studien um Blockaden des Plexus brachialis (axilläre [6 Studien], infraklavikuläre [6], interskalenäre Technik [3]), in 7 Studien um Ischiadicusblockaden und in 1 um eine Medianus-Ulnaris-Blockade. Die zu blockierenden Nerven waren dabei per Ultraschall, mit elektrischer Stimulation oder einer Kombination aus beidem aufgesucht worden.

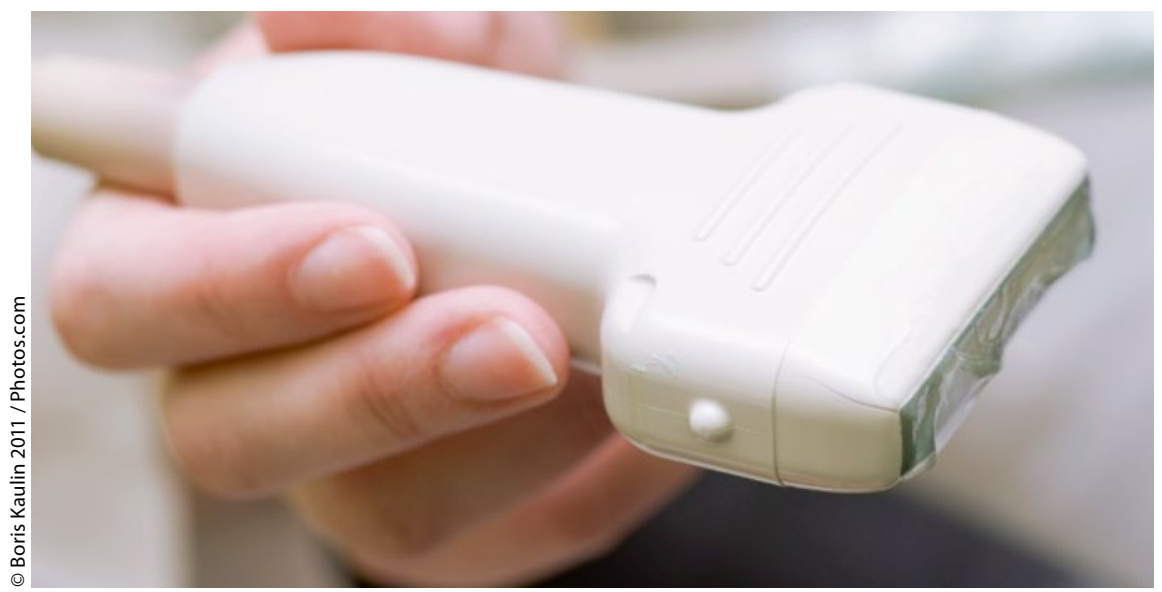

Die Ultraschalltechnik führte zu einem Paradigmenwechsel bei der Visualisierung von Nervenstrukturen zur Regionalanästhesie.
Die Zahl von Patienten, die Schmersank nach sonografischer Nervensuche, verglichen mit elektrischer Stimulation, im Mittel um $40 \%$. Die Rate zusätzlich nötiger Analgesie- bzw. Anästhesiemaßnahmen ging um $60 \%$ zurück. Und die Zahl versehentlich angestochener Gefäße lag um $77 \%$ niedriger.

Außerdem setzte die sensorische Blockade nach sonografisch geführter Injektion rascher ein, ohne dass bei den postoperativen neurologischen Nebenwirkungen ein Unterschied festzustellen gewesen wäre. Den Ultraschall mit elektrischer Stimulation $\mathrm{zu}$ kombinieren, verbesserte die Ergebnisse nicht weiter. zen während der Prozedur verspürten,
Im rechnerischen Vergleich zur elektrischen Stimulation erhöhte das Ultraschallverfahren die Zahl erfolgreicher peripherer Nervenblockaden von 828 auf 918 je 1.000 Patienten. Gleichzeitig sank die Rate von Patienten, die einer weiteren Analgesie, Sedierung oder Anästhesie bedurften, von 172 auf 82 je 1.000 Patienten.

Fazit: Munirama und McLeod schlagen vor, sich in künftigen Vergleichsstudien zur Ortung von Schmerzen mit Ultraschall bzw. elektrischer Stimulation auf weitere Aspekte zu konzentrieren, etwa die Differenzen in der langzeitigen Schmerzlinderung, der Patientenzufriedenheit, der Gliedmaßenfunktion, bei den stationären Wiederaufnahmen, unerwünschten Effekten und den Kosten. In der seit Januar 2015 und noch bis 2020 gültigen deutschen Leitlinie „Handlungsempfehlung zur Nervenlokalisation für periphere Regionalanästhesieverfahren" ist zu lesen, die Einführung der Sonografie habe zu einem $\mathrm{Pa}$ radigmenwechsel geführt.

Die elektrische Nervenstimulation wird von den Autoren - federführend ist die Deutsche Gesellschaft für Anästhesiologie und Intensivmedizin (DGAI) aber keineswegs verworfen. „Es ist festzuhalten“, schreiben sie, „dass die elektrische Nervenstimulationstechnik nach wie vor zur Nervenlokalisation ohne parallele Verwendung der Sonografie eingesetzt werden kann, ohne damit gegen die Regeln der ,Good Clinical Practice zu verstoßen.“

Robert Bublak

Munirama S, McLeod G. A systematic review and meta-analysis of ultrasound versus electrical stimulation for peripheral nerve location and blockade. Anaesthesia. 2015;70(9):1084-91. 